Supporting Information

\title{
Overcurrent electrodeposition of fractal plasmonic black gold with broadband absorption for excitation-immune SERS
}

Renpeng Yu,,$^{\dagger}$ Jingyu Wang,,$^{\dagger}$ Mei Han,$^{\dagger}$ Mengyao Zhang, ${ }^{\dagger}$ Pei Zeng,,$\S$ Weiqi Dang,,$\succ$

Jianfang Liu, ${ }^{\dagger}$ Zhilin Yang, ${ }^{,+}$Jiawen Hu, ${ }^{,+\dagger}$ and Zhongqun Tian ${ }^{\&}$

†State Key Laboratory for Chemo/Biosensing and Chemometrics, Hunan Key Laboratory of Two-Dimensional Materials, and College of Chemistry and Chemical Engineering, Hunan University, Changsha 410082, China

\#Department of Physics, Research Institute for Biomimetics and Soft Matter, Xiamen University, Xiamen 361005, China

§School of Physics and Electronics, State Key Laboratory of Advanced Design and Manufacturing for Vehicle Body, Hunan University, Changsha 410082, China

\&State Key Laboratory for Physical Chemistry of Solid Surfaces and Department of Chemistry, Xiamen University, Xiamen 361005, China

\section{Supplementary Figures}
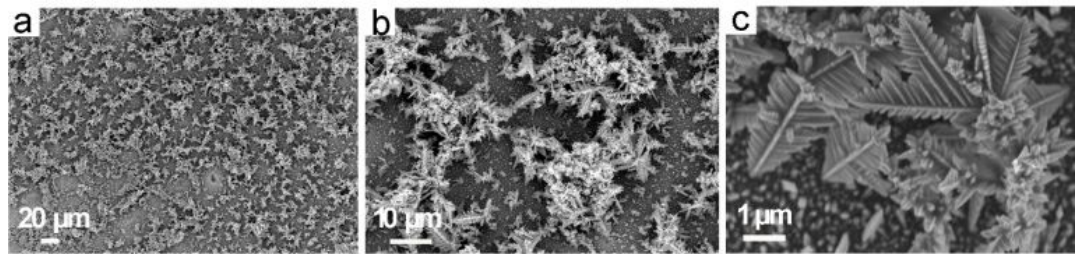

Figure S1. (a-c) SEM images for the B-Au with different magnifications. 

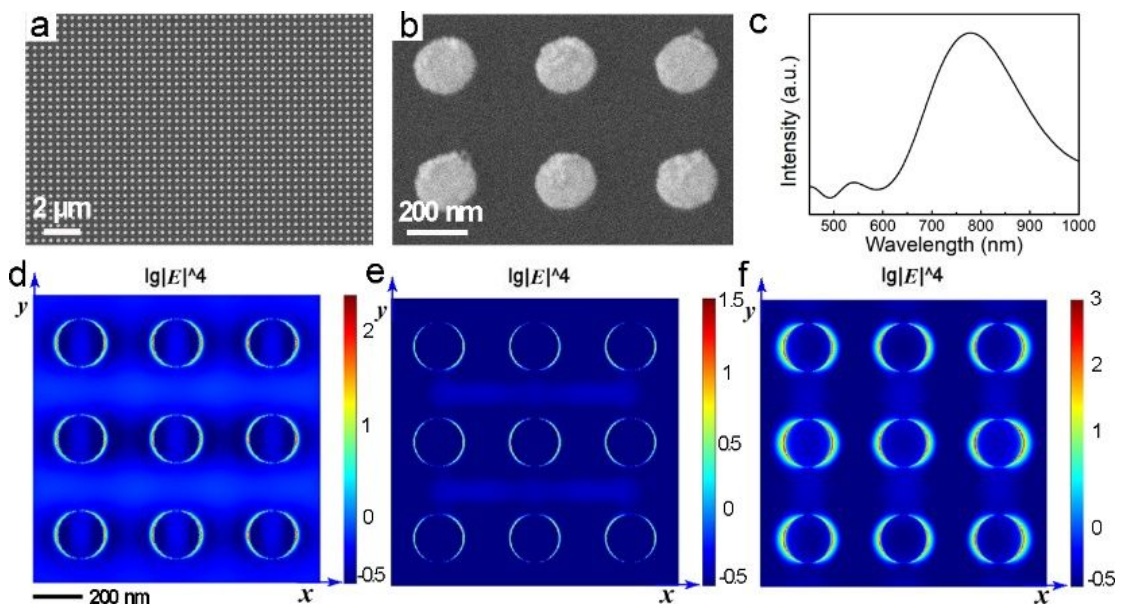

Figure S2. (a, b) SEM images for Au nanodisk array with different magnifications and (c) corresponding FDTD-simulated scattering spectrum; field distributions on $\mathrm{Au}$ nanodisk excited at (d) 532, (e) 633 (f) $785 \mathrm{~nm}$ simulated using 3D-FDTD method.

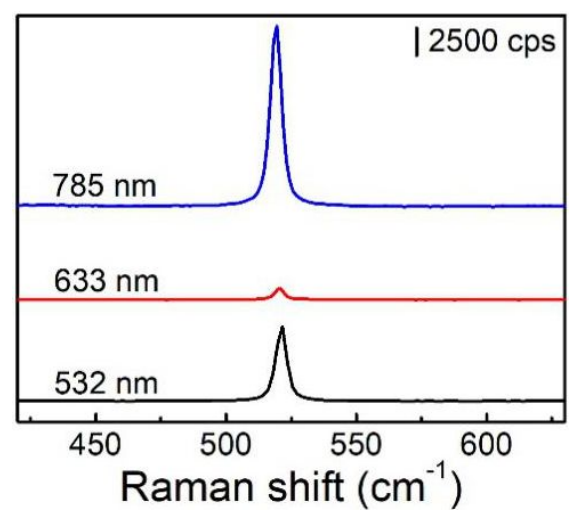

Figure S3. Raman spectra of silicon wafer excited using 532, 633, and $785 \mathrm{~nm}$ laser for a single 1-s accumulation with the power of each laser attenuated to $10 \%$.
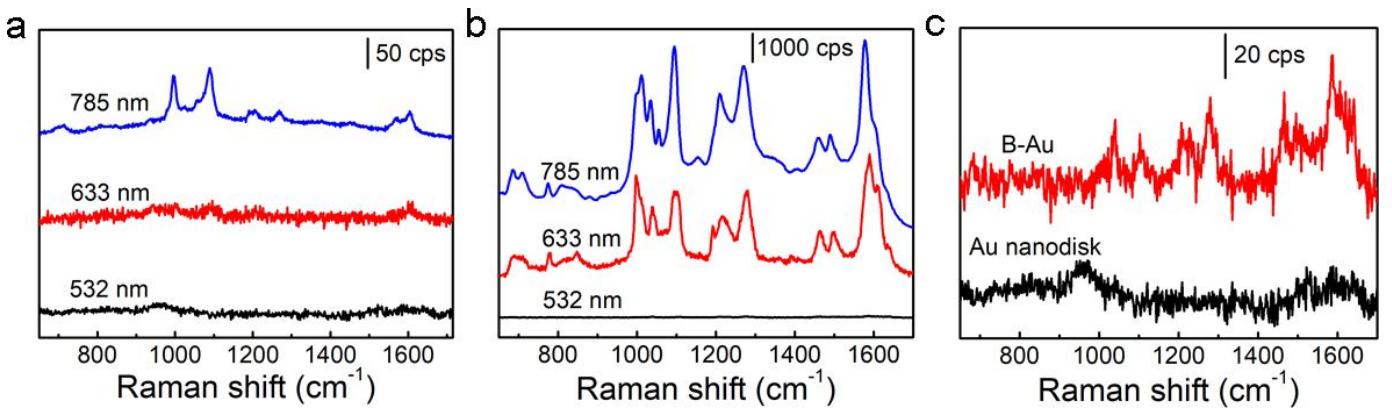

Figure S4. Normalized SERS spectra of 4-MPY on (a) Au nano-disk array and (b) $\mathrm{B}-\mathrm{Au}$ at different excitation and (c) SERS spectra of 4-MPY on Au nano-disk and 
B-Au at $532 \mathrm{~nm}$ excitation. 